\title{
REPORTE DEL HALLAZGO DE ÁRBOLES CULTURALMENTE MODIFICADOS EN BOSQUES COSTEROS DE NOTHOFAGUS BETULOIDES (MIRB.) OERST 1871 (NOTHOFAGACEAE) POR PUEBLOS CANOEROS DE LA PATAGONIA AUSTRAL Y TIERRA DEL FUEGO.
}

\section{GABRIEL ZEGERS*, MACARENA FERNÁNDEZ ${ }^{\star *}$, BENJAMÍN CÁCERES***,

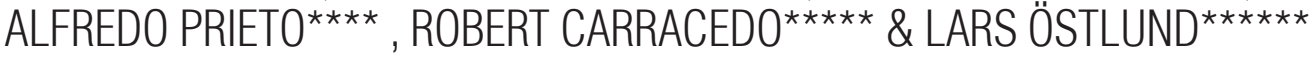

\section{Introducción}

Los bosques templados costeros pluviales de la Patagonia austral y Tierra del Fuego constituyen una expresión florística y ecológica de características propias y muy distintas al resto de las formaciones vegetales del continente americano (Pisano, 1997). Aquellos de carácter "primario" o en estados sucesionales avanzados son actualmente remanentes escasos de los procesos antrópicos de degradación ambiental relacionados a la extracción y explotación de recursos naturales, que sobreviven gracias a su aislamiento de caminos o rutas de explotación, o bien, por su resguardo geográfico a eventos climáticos de gran envergadura (i.e. volteo masivo por temporales de viento) (Pisano, 1997; Promis, Cruz, Reif \& Gärtner, 2008). Conservan en su estructura informa- ción de enorme y singular valor ecológico, siendo testigos vivos de procesos ambientales y paleoclimáticos, estrechamente ligados a las dinámicas históricas del paisaje litoral de los canales patagónicos (Holz, Haberle, Veblen, De Pol Holz, Southon, 2012; Koch \& Kilian, 2002; Villagrán, 2018). A su vez, es de esperar, que dichas dinámicas podrían comprender en parte o en su totalidad, una historia estrechamente ligada al ser humano, al menos desde el retroceso glacial y posterior ocupación de los canales patagónicos por cazadores recolectores marinos o pueblos canoeros (Emperaire, 1955). De esta manera, es posible inferir que muchos de estos ecosistemas, conservan en su estructura parte del legado cultural de uso y transformación del bosque por las poblaciones ancestrales de canoeros kawésqar y yagan que poblaron estas latitudes desde hace 6.000 a 7.000 años atrás

\footnotetext{
*MSc. en Recursos Naturales. Asociación de Investigadores del Museo de Historia Natural Río Seco, Punta Arenas, Chile. Correo-e: gabrielzegersm@gmail.com

**Antropóloga. Centro de Estudios del Cuaternario Fuego Patagonia y Antártica Chilena (Fundación CEQUA), Punta Arenas, Chile.Correo-e: macarena.fernandez@cequa.cl

***Bologo Marino. Asociación de Investigadores del Museo de Historia Natural Río Seco, Punta Arenas, Chile. Centro de Estudios del Cuaternario Fuego Patagonia y Antártica Chilena (Fundación CEQUA), Punta Arenas, Chile. Correo-e: benjamincaceresm@gmail.com

${ }_{\star \star \star \star}$ Dr. en Arqueología. Universidad de Magallanes, Centro Puerto Natales, Chile. Correo-e: alfredo.prieto@umag.cl ***** Universitat Autònoma de Barcelona, Departament de Prehistòria, España. Correo-e: robert.carracedo@e-campus.uab.cat ***** Dr. en Ecología Forestal. Department of Forest Ecology and Management, Swedish University of Agricultural Sciences (SLU), Umeå, Suecia. Correo-e: Lars.Ostlund@slu.se
} 
(Legoupil \& Fontugne, 1997). La presente nota tiene por objetivo describir el hallazgo reciente de árboles con marcas culturales (i.e. antiguas cicatrices de descortezamiento), registrados en diversos puntos del territorio de fiordos y canales de la Patagonia occidental, y con ello aportar nuevos antecedentes sobre el uso ancestral de los bosques costeros de Nothofagus betuloides.

\section{Área de estudio}

El área de estudio de este trabajo comprende el territorio que abarca desde el golfo de Penas hasta Cabo de Hornos, incluyendo las costas occidentales del estrecho de Magallanes y parte del archipiélago de Tierra del Fuego. Es un sistema complejo de cuencas hidrográficas sumergidas que forman parte de la cordillera de los Andes, modeladas durante el Holoceno temprano por el avance y retroceso glaciar, dando lugar a la actual trama de islas, canales, ensenadas y fiordos que componen el paisaje litoral (Pisano, 1997).

Las condiciones climáticas son muy variadas desde las costas continentales hacia el oeste de la cordillera andina en el océano Pacífico, con precipitaciones anuales que oscilan entre los $400 \mathrm{~mm}$ y los $7.000 \mathrm{~mm}$ (Schneider, Glaser, Kilian, Santana, Butorovic \& Casassa, 2003). De igual manera varía su biogeografía, pasando de una delgada franja transicional de praderas húmedas y bosques de Nothofagus antártica, Nothofagus pumilio y Nothofagus betuloides, a un paisaje de canales expuestos al viento con poca cobertura arbórea compuesta principalmente, en las zonas intermedias o bajas, por turberas del musgo Sphagnum sp. De manera más aislada y limitados a los sitios costeros protegidos de los fuertes vientos, como bahías o fiordos, se encuentran los pisos vegetacionales de bosque siempreverde templado costero de Nothofagus betuloides y Drimys winteri y/o Embothrium coccineum (Luebert \& Pliscoff, 2006). Especialmente para efectos de este trabajo, destacan los llamados bosques mixtos de $N$. betuloides, $D$. winteri y Pseudopanax laetevirens, ubicados bajo los 100 m.s.n.m.. Se caracterizan por sus suelos orgánicos con acumulación de turba en el primer horizonte y cubiertos por una gruesa capa de detritus de hojarasca y madera en distintos estados de descomposición (Pisano, 1997; Promis et al., 2008). Estos ecosistemas, situados en bahías y riberas protegidas comúnmente asociadas a recursos marinos adyacentes, forman parte fundamental del nicho ecológico de las poblaciones canoeras kawésqar y yagan de los fiordos y canales de la Patagonia austral (Emperaire, 1955).

\section{Contexto del hallazgo}

En el marco de un proyecto de investigación para la identificación y análisis de las dinámicas de estructura de ecosistemas forestales costeros de alto valor ecológico y cultural, se ha podido observar de manera preliminar, en tres campañas de terreno durante los años 2017 y 2018, descortezamientos que podrían indicarnos un uso de larga data por parte de pueblos canoeros australes. Los descortezamientos han sido encontrados en diversos puntos de la Patagonia austral (fig. 1), en bosques costeros ubicados en bahías protegidas; uno de estos (río Batchelor, fig. 1) en el contexto de un sitio arqueológico estudiado anteriormente por Legoupil et al. (2014). Cabe destacar que muchos de estos árboles con cicatrices de descortezamiento presentan marcas de un 
posible uso de herramientas cortantes utilizadas para tal caso.

Utilizando recursos como documentos de viajes exploratorios y expediciones científicas de los siglos XVII, XVIII y XIX, junto con estudios arqueológicos, etnográficos y comunicaciones personales de pescadores, se están identificando sitios de interés en las costas del Área Marina Protegida Francisco Coloane en el estrecho de Magallanes, así como en el golfo Almirante Montt, provincia de Última Esperanza y en las costas del Parque Nacional Alberto De Agostini (fig. 1), en la cordillera Darwin. Actualmente, el trabajo se ha centrado principalmente en la identificación de ecosistemas forestales de alto valor cultural, así como en el análisis de las dinámicas sucesionales de sitios de valor arqueológico.

Fig. 1. Sitios (puntos de colores) que han sido visitados y estudiados hasta la fecha por el presente equipo de investigación.

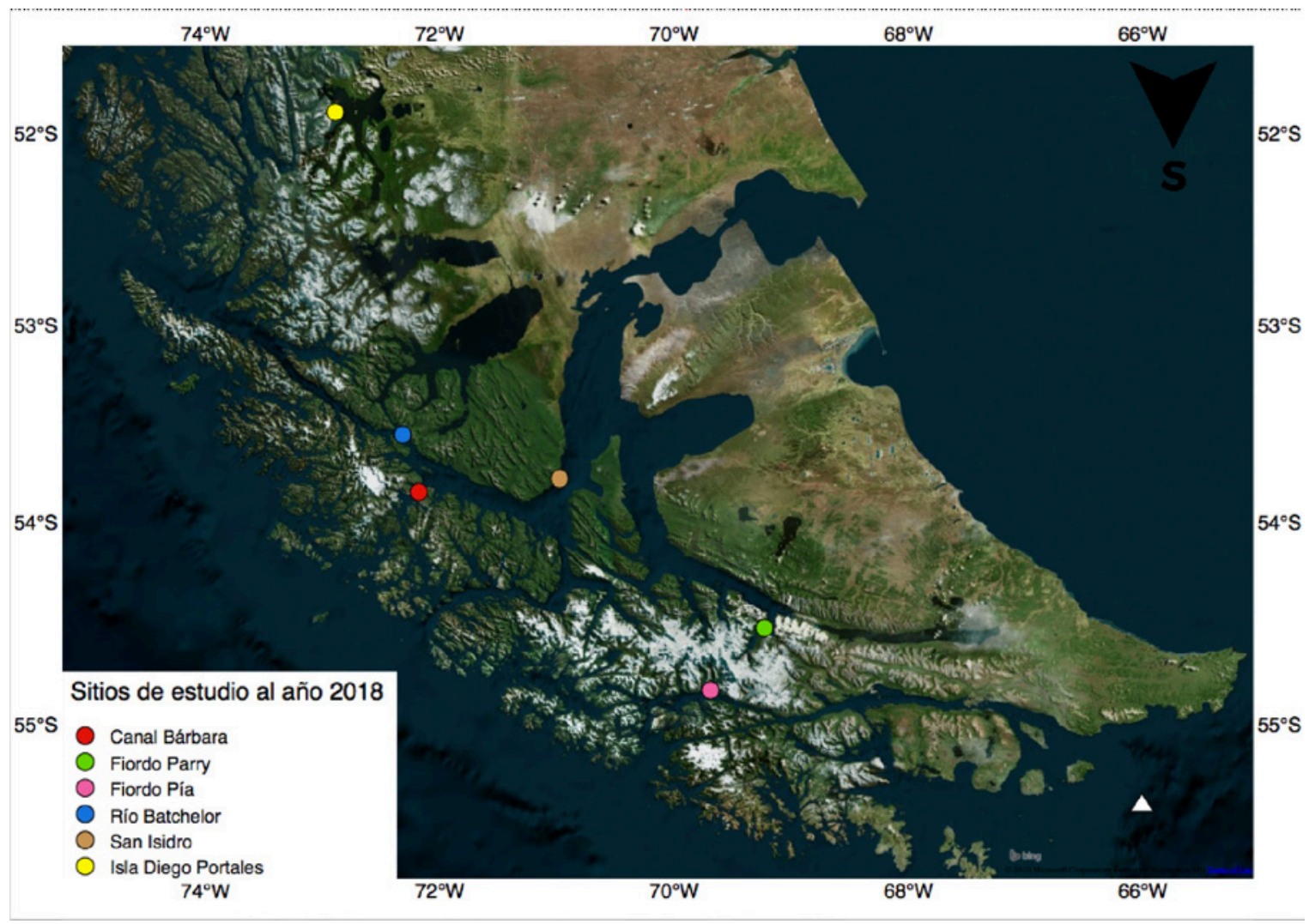

Fuente: Elaborado por Gabriel Zegers en base a Imagen de Bing Maps 2019. 


\section{Referencias históricas al uso de la corteza y descripción del hallazgo}

El uso de los recursos naturales y la transformación del paisaje por poblaciones canoeras de la Patagonia austral y Tierra del Fuego ha sido descrito por navegantes, misioneros, etnógrafos, historiadores, antropólogos y arqueólogos, entre otros, y en diversas expediciones exploratorias y científicas, dentro de las que destacan trabajos como los de Emperaire (1955), Gusinde (1986) y Chapman (2012). De los árboles se extraía la corteza, un recurso fundamental para la construcción de sus campamentos, para la fabricación de recipientes (fig. 2) y para la construcción de canoas (Emperaire, 1955). Es sabido también que con la corteza confeccionaban máscaras utilizadas en rituales de iniciación (Gusinde, 1986) e incluso se sonsumía su savia (Martínez-Corvetto, 1968; Zárraga, 2017). De acuerdo a estos autores, las especies de árboles utilizadas para la elaboración de artículos de corteza eran el coigüe ( $N$. betuloides), el canelo (D. winteri), el maitén (Maytenus magellanica), el tenío (Weinmannia trichosperma) y el mañiu (Podocarpus nubigena), estos dos últimos restringidos biogeográficamente a la zona norte del área de estudio. Para el consumo alimenticio de sustancias dulces de la savia se utilizaba la lenga (N. pumilio) (Zárraga, 2017) y el coigüe (N. betuloides) (com. pers. de Gabriela Paterito). Los registros existentes, así como la propia fisiología de estas especies, coinciden en que la extracción de corteza era realizada al comienzo de la primavera, cuando los árboles movilizan carbohidratos y nutrientes por el floema desde el sistema radical hacia las ramas superiores para el desarrollo del follaje. La extracción era realizada mediante herramientas cortantes como conchas afiladas y posteriormente con latas o metales afilados, para luego separar la corteza mediante descortezadores de hueso de guanaco o trozos de hueso de ballena labrados en forma de cuña (Orquera \& Piana, 1999).

Fig. 2. Recipiente fabricado de corteza (posiblemente Drimys winteri) y fibras vegetales llevado por C. Skottsberg al Museo Etnográfico Gotemburgo, Suecia.
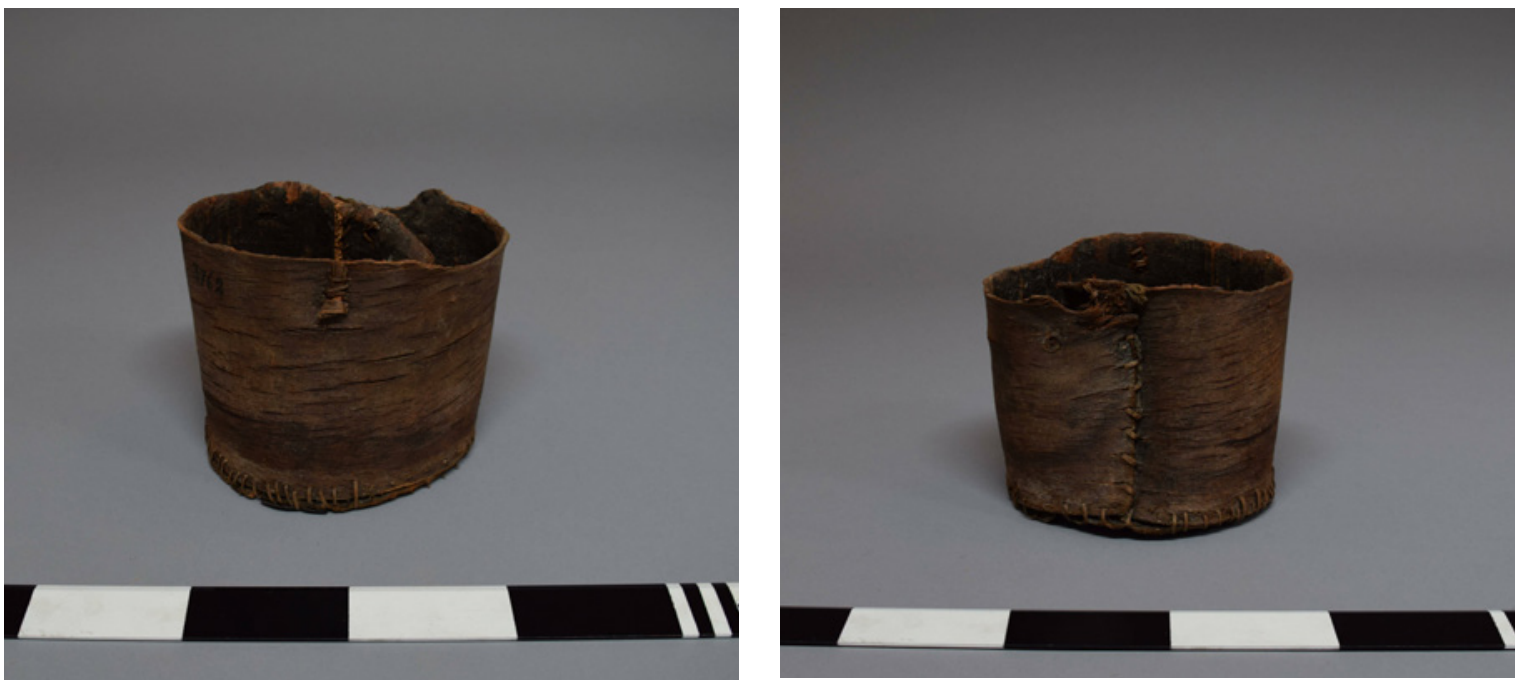

Fotografía: Benjamín Cáceres. 
El hallazgo consiste en árboles de $N$. betuloides, vivos y muertos, los cuales presentan cicatrices de descortezamiento parcial respecto a la circunferencia del tronco, de forma rectangular o triangular, con dimensiones que varían entre los $10 \mathrm{~cm}$ y los $25 \mathrm{~cm}$ de ancho (dependiendo cuan antigua y cerrada está la cicatriz) y los 50 $\mathrm{cm}$ a $200 \mathrm{~cm}$ de largo. Todas tienen en común que su base se encuentra por sobre la base del árbol y no a ras de suelo, como suelen presentarse las cicatrices de fuego en árboles afecta- dos por incendios. Algunos de ellos presentan marcas o incisiones claras que sugieren el uso de herramientas cortantes o cuñas para deslizar entre la corteza interna (floema) y el xilema (fig. 3 y 4). Cabe destacar que a la fecha solo existe un registro fotográfico (fig. 4, 5 y 6) de los árboles culturalmente modificados evidenciados en terreno, sin embargo el presente equipo está trabajando por la datación dendrocronológica y registro sistemático de las dimensiones de estas cicatrices.

Fig. 3. Ilustración realizada en la desembocadura del río Batchelor de un árbol culturalmente modificado, con cicatriz de descortezamiento y claras marcas del uso de cuña para desprender la corteza del xilema del árbol.

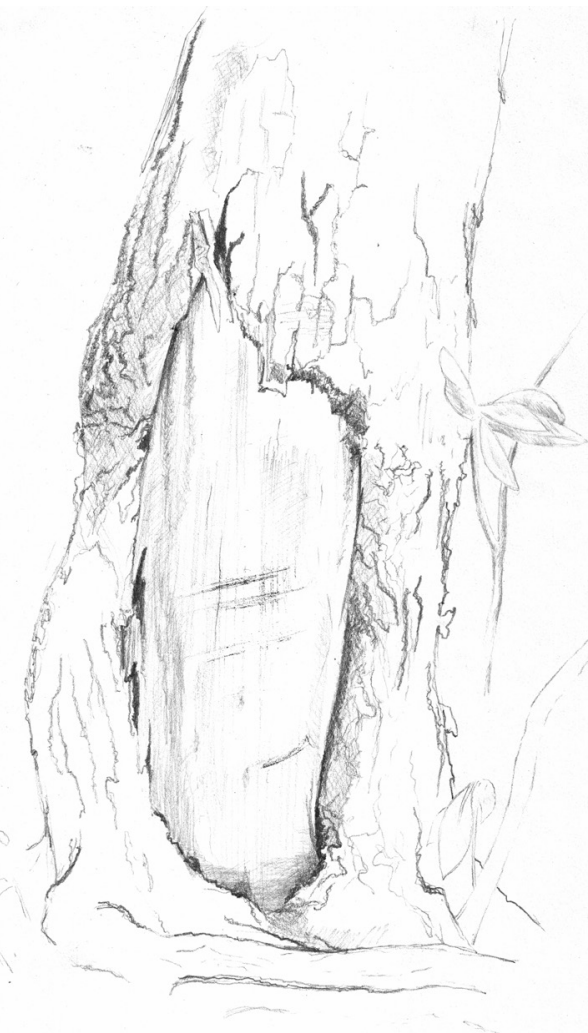

Autor: Miguel Cáceres Murrie. 
Fig. 4. Nothofagus betuloides con cicatrices de descortezamiento mediante herramienta cortante, punta Barría, fiordo Parry, Parque Nacional Alberto de Agostini.

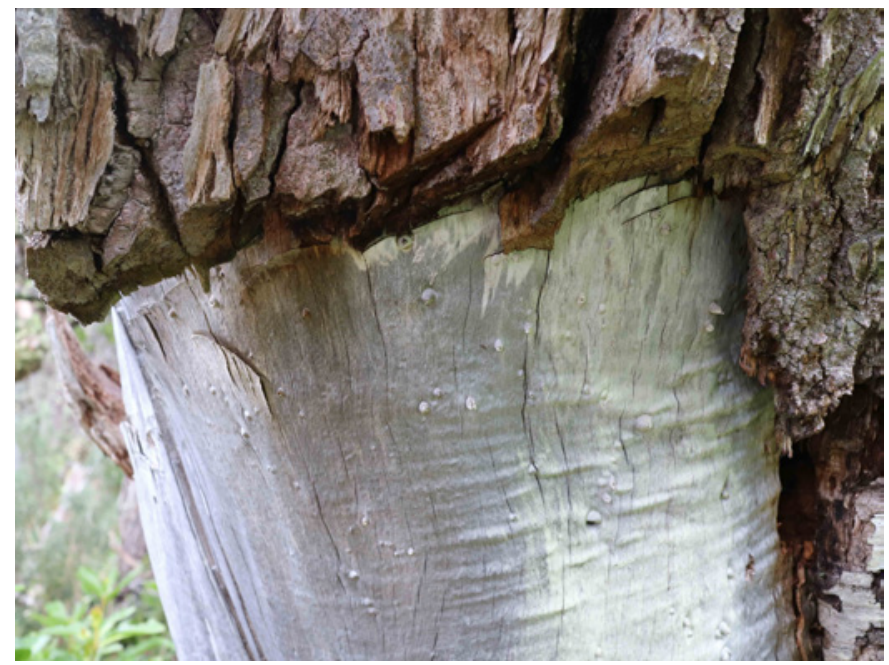

Fotografía: Lars Östlund.

Fig. 5. Árbol vivo con cicatriz de descortezamiento en fiordo Pía (izquierda) y árbol muerto con cicatriz de descortezamiento y marcas de herramienta antrópica en río Batchelor (derecha).
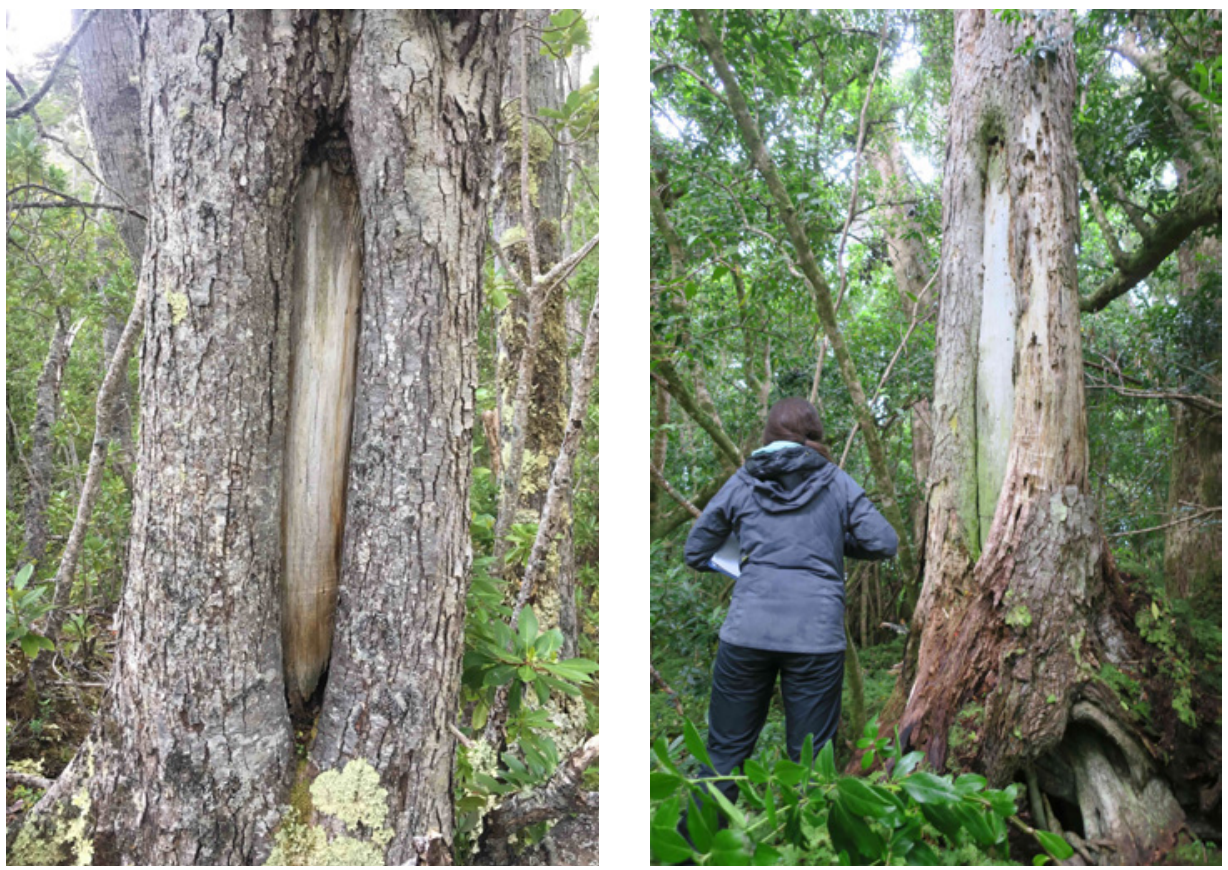

Fotografías: Gabriel Zegers (izquierda) y Robert Carracedo (derecha). 


\section{Ejemplos y casos de estudio en otras latitudes}

Múltiples líneas de investigación en el hemisferio norte, especialmente en las zonas boreales del norte de Escandinavia, Siberia y Norte América, han demostrado mediante evidencias que los ecosistemas forestales son testigos materiales vivos de la historia natural de los humanos (Mobley \& Eldridge, 1992; Östlund, Keane, Arno \& Anderson, 2005; Rautio, Josefsson, Axelsson \& Östlund, 2016; Turner, Ari, Berkes, Davidson Hunt, Ertug \& Miller, 2009). A través de herramientas y métodos de la ecología histórica, pruebas arqueológicas y descripciones etnográficas del territorio, se ha logrado evidenciar el uso y manejo histórico de bosques, así como identificar rangos de ocupación de un territorio forestal, intensidad, periodicidad de uso y forma de extracción de los recursos. De acuerdo a estos trabajos, los elementos que para fines históricos constituyen el testigo de mayor precisión temporal, son los árboles (Östlund, Ahlberg, Zackrisson, Bergman \& Arno, 2009). Su ciclo de desarrollo anual o temporal en los anillos de crecimiento permite datar de manera exacta o estimada la ocurrencia de una marca o alteración, ya sea de origen natural o antrópico. Interesan en este caso aquellas realizadas por humanos en el pasado, como por ejemplo, cortes o podas en los árboles y cicatrices de descortezamiento, las que mediante muestras dendrocronológicas podrían ser fechadas e interpretadas dentro de un contexto histórico (Östlund, Zackrisson \& Hörnberg, 2002). Estos registros son conocidos internacionalmente como "marcas culturales" y aquellos árboles que las poseen, como "árboles culturalmente modificados" (en inglés "culturally modified trees") (Mobley \& Eldridge, 1992).

La similitud, tanto en la forma de los descortezamientos, como en la presencia de marcas de corte o golpe con herramientas sobre la madera expuesta de árboles antiguos registrados en los canales patagónicos, con los estudios realizados sobre árboles culturalmente modificados del hemisferio norte (fig. 6), llama de gran manera la atención. Sin embargo, a pesar de la presencia de árboles descortezados en bosques maduros o "primarios" de los canales, especialmente aquellos ligados a un contexto arqueológico, se ha evidenciado la dificultad para determinar de manera certera el hecho de que un árbol sea culturalmente modificado, especialmente en aquellos casos en que la madera expuesta se encuentra degradada haciendo imposible la observación de marcas de corte. En este caso, la evidencia anteriormente mencionada en bosques boreales del hemisferio norte, así como la interpretación mediante diversas herramientas (i.e. dendrocronología) del contexto local, llaman a realizar estudios profundos y acabados sobre las características principales de los árboles culturalmente modificados, las técnicas utilizadas para su modificación y el análisis de patrones estructurales de estas marcas en el contexto local. 
Fig. 6. Fotografías de un árbol culturalmente modificado a orillas del río Batchelor, Chile (izquierda) y en la Reserva Natural de Tjegglevas, Suecia (derecha).
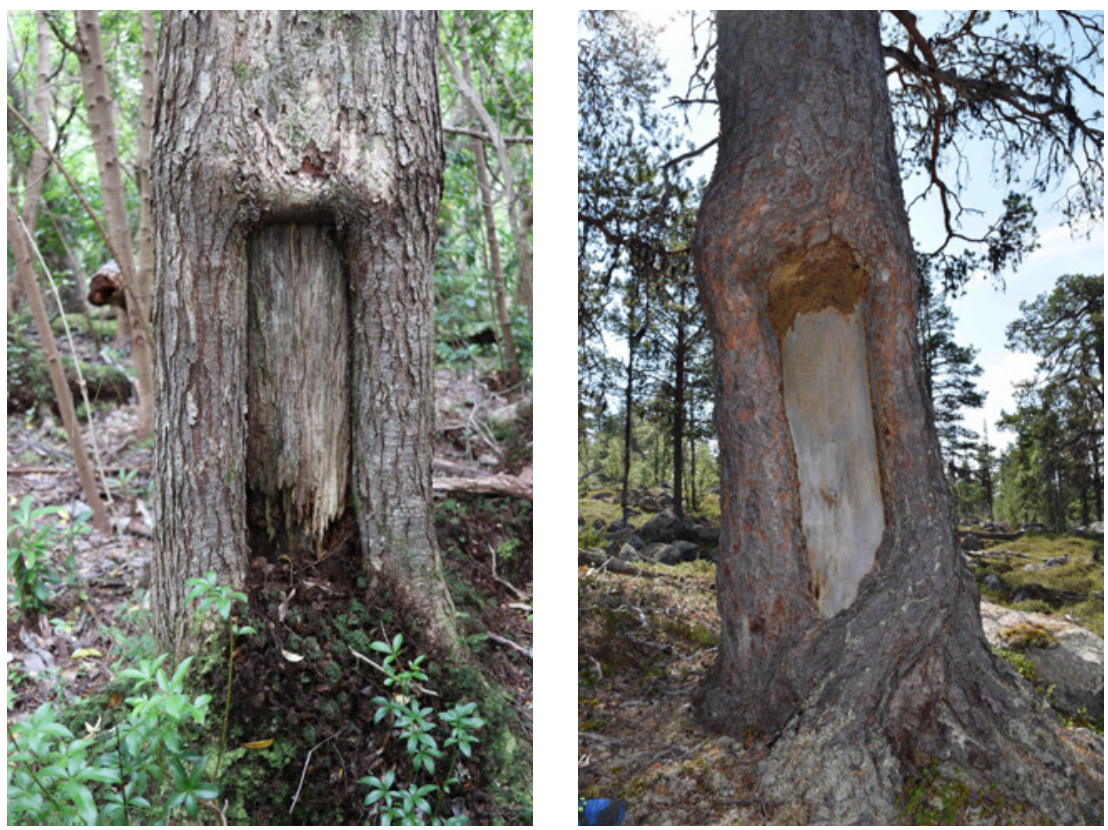

Fotografías: Lars Östlund (izquierda) y Benjamín Cáceres (derecha).

\section{Conclusión}

Los autores destacan la importancia de continuar y fortalecer estudios que permitan levantar y disponer de mayor información sobre la temática en cuestión, desde una perspectiva territorial. Existen desafíos particulares ligados al estudio de los árboles descortezados en la región. Los árboles antiguos pueden recibir otras fuentes (no antrópicas) causantes de daño sobre la corteza, como la caída de otros árboles y ramas por temporales de viento. Así también la relativamente rápida descomposición de la superficie descortezada y la madera interna del árbol sugieren otro problema metodológico. Ambos factores constituyen un desafío para determinar el origen de las cicatrices, por causas naturales o antrópicas. Es necesario desarrollar estándares y nuevas metodologías para futuros estudios.

Más aún, se destaca la gran importancia de integrar el estudio de la singularidad ecológica de los bosques templados costeros pluviales de la Patagonia austral y Tierra del Fuego, con el legado cultural centenario que estos conservan y el valor patrimonial que representan, generando nuevas preguntas, métodos y acciones para asegurar su conservación para las presentes y futuras generaciones. Si bien actualmente muchos de estos bosques se encuentran bajo alguna categoría de protección, ya sea dentro del Sistema Nacional de Áreas Protegidas del Estado o meramente protegidos por la ley 20.28 sobre Recuperación del Bosque Nativo y Fomento Forestal del Ministerio de Agricultura, no se hace referencia 
explícita en el sistema legal chileno a los árboles culturalmente modificados y la importancia de su conservación. Es de esperar que futuros esfuerzos sirvan para crear nuevas herramientas que se encaminen hacia las políticas públicas en cuanto a la protección y conservación tanto de estos ecosistemas como del legado cultural de los pueblos canoeros y aquellas comunidades indígenas que son hoy depositarias de dicho legado y valores patrimoniales.

\section{Referencias bibliográficas}

Chapman, A. (2012). Yaganes del Cabo de Hornos. Encuentros con los europeos antes y después de Darwin. Santiago: Liberalia - Pehuen Editores.

Emperaire, J. (1955). Les Nomads de la Mer. Paris: Gallimard.

Gusinde, M. (1986). Los indios de Tierra del Fuego, Tomo I, II y III, Los Selk'nam, los Yámana, los Halakwulup. Buenos Aires: Centro Argentino de Etnología Americana.

Holz, A., Haberle, S., Veblen, T. T., De Pol-Holz, R. \& Southon, J. (2012). Fire history in western Patagonia from paired tree-ring fire-scar and charcoal records. Climate of the Past, 8(2), 451-466.

Koch, J. \& Kilian, R. (2002). Potencial dendrocronológico de especies de árboles típicos en una transecta sobre los Andes australes de Chile (53 S). Anales Del Instituto de La Patagonia, 30, 123-132.

Legoupil, D., Christensen, M., Laporal, D., De Miranda, I., Morello, F., Pellé, E., San Román, M. \& Teyssandier, J. (2014). Le parc Marin Coloane: prospection archéologique. Rapport au ministère des Affaires Etrangères, France. París.

Legoupil, D. \& Fontugne, R. (1997). El poblamiento maritimo en los archipielagos de la Patagonia: nucleos antiguos y dispersión reciente. Anales Del Instituto de La Patagonia, 25, 75-87.

Luebert, F. \& Pliscoff, P. (2006). Sinópsis Bioclimática y Vegetacional de Chile. Santiago, Chile: Editorial Universitaria.

Martínez-Corvetto, R. (1968). Estudios Etonotánicos: Nombre de plantas y su utilidad según los indios Onas de Tierra del Fuego. Etnobiológica. Facultad de Agronomía y Veterinaria. Universidad Nacional del Norte.

Mobley, C. \& Eldridge, M. (1992). Culturally modified trees in the Pacific Northwest. Arctic Anthropology, 29(2), 91-110.

Orquera, L. A. \& Piana, E. L. (1999). La vida material y social de los Yámana (1st ed.). Buenos Aires: Eudeba.

Östlund, L., Ahlberg, L., Zackrisson, O., Bergman, I. \& Arno,

\section{Agradecimientos}

Los autores agradecen a Liz y Gifford Hickey de Springcreek Conservation y a la Swedish University of Agricultural Sciences (SLU) por el fianciamiento que hizo posible las campañas de terreno; a la tripulación de la Marypaz II por su voluntad y disposición en las campañas de muestreo, a Torbjörn Josefsson y Samuel Roturier por su apoyo intelectual y en terreno; a Miguel Cáceres por su apoyo intelectual y artístico con la ilustración del árbol descortezado; a Gabriela Paterito e Isabel Tonko por su generosidad en conversaciones y su enérgico apoyo en terreno.

S. (2009). Bark-peeling, Food Stress and Tree Spirits - the Use of Pine Inner Bark for Food in Scandinavia and North America. Journal of Ethnobiology, 29(1), 94-112.

Östlund, L., Keane, B., Arno, S. \& Andersson, R. (2005). Culturally scarred trees in the bob marshall wilderness, Montana, USA - interpreting native american historical forest use in a wilderness area. Natural Areas Journal, 25, 315-325.

Östlund, L., Zackrisson, O. \& Hörnberg, G. (2002). Trees on the border between nature and culture: culturally modified trees in boreal Sweden. Environmental History, 7(1), 48-68.

Pisano, E. (1997). Los bosques de la patagonia austral y tierra del fuego chilenas. Anales Del Instituto de La Patagonia, 25(19), 10.

Promis, A., Cruz, G., Reif, A. \& Gärtner, S. (2008). Nothofagus Betuloides (MIRB) Oerst 1871 (Fagales: Nothofagaceae) Forests in Southern Patagonia and Tierra del Fuego. Anales Del Instituto de La Patagonia, 36, 53-68.

Rautio, A. M., Josefsson, T., Axelsson, A. L. \& Östlund, L. (2016). People and pines 1555-1910: integrating ecology, history and archaeology to assess long-term resource use in northern Fennoscandia. Landscape Ecology, 31(2), 337-349.

Schneider, C., Glaser, M., Kilian, R., Santana, A., Butorovic, N. \& Casassa, G. (2003). Weather Observations Across the Southern Andes at 53 S. Physical Geography, 24(2), 97-119.

Turner, N. J., Ari, Y., Berkes, F., Davidson-Hunt, I., Ertug, Z. F. \& Miller, A. (2009). Cultural management of living trees: an international perspective. Journal of Ethnobiology, 29(2), 237-270.

Villagrán, C. (2018). Biogeografía de los bosques subtropicaltemplados del sur de sudamérica. Hipótesis históricas. Magallania, 46(1), 27-48.

Zárraga, C. (2017). Cristina Calderón. Memorias de mi abuela Yagan. (O. Vogles, Ed.). Punta Arenas: Ediciones PIX. 